\title{
Etude de mélanges vases-ciment en vue d'une réutilisation éventuelle en chantier d'injection
}

\author{
Jean-Pierre Troalen*, Vincent Lobry et Elodie Scheller** \\ * Maître de Conférences, INPL-ENSG, Laboratoire Environnement, \\ Géomécanique \& Ouvrages, ** Elèves-ingénieurs ENSG.
}

Résumé

L'éventuelle utilisation en chantier d'injection sous forme de coulis vases/ciment de sédiments dragués les plus fins nécessite une caractérisation préalable : principalement minéralogie, dimension des constituants solides, plasticité et densité. L'étude du comportement mécanique des mélanges vasesciment (évaluation de la réaction de prise) a été abordé dès la réalisation des mélanges par la mesure de la cohésion intacte non drainée au scissomètre, puis par la mesure de la résistance en compression uniaxiale sur les éprouvettes fabriquées à partir des mélanges et après un repos d'une durée de 7 jours. Cette étude a permis d'établir un mode opératoire spécifique et de définir les paramètres nécessaires en vue de la réutilisation éventuelle de vases en chantier d'injection.

\section{Abstract}

The potential utilization of muds-cement mixtures for jet-grouting is conceivable with a preliminary physico-chemical characterization : mineralogy, size of solid particles, plasticity and density. The mechanical behaviour has been studied in scissometer test (natural undrained cohesion) and in uniaxial compression test for different muds/cement mixtures after seven days. This study has allowed to establish a specific proceeding. The necessary parameters for a potential utilization in jet-grouting of these muds/cement mixtures are definited.

\section{Introduction}

Dans le cadre d'études actuelles sur le devenir des vases et des boues issues du dragage, il est envisagé diverses utilisations éventuelles. En collaboration avec le professeur C. Louis de l'université du Havre, nous étudions les possibilités d'introduction de sédiments fins comme support du ciment dans les coulis d'injection. Le comportement de tels coulis repose, d'une part sur la composition minéralogique et granulométrique du support, et d'autre part sur la densité et la 
composition des coulis (proportions relatives entre support et ciment). Comme dans toute étude sur le devenir des matériaux issus du dragage, il convient d'en effectuer au préalable la caractérisation pétrophysique (Marot, 1997 ; Troalen, 1996). Il est primordial dans le cas présent de définir la densité initiale du matériau support, l'objectif étant bien entendu de pouvoir l'utiliser directement dans son état physique après dragage. Ces paramètres vont nous permettre de préparer selon un mode opératoire les différents coulis vases-ciment et de réaliser les éprouvettes sur lesquelles sera effectuée l'étude du comportement mécanique. En effet, les quantités respectives utilisées dans la réaction de prise sont conditionnées par la composition minéralogique et dimensionnelle du support et par les proportions adoptées pour le mélange, c'est-à-dire la fabrication du coulis d'injection. La plus forte résistance obtenue correspondra, dans ce cas, à un optimum ciment-support où un maximum de liaisons fortes aura été ainsi créé. Une injection a en effet pour but aussi bien l'amélioration des propriétés mécaniques d'un massif que celles d'un ancrage.

\section{Caractéristiques physiques des vases}

Les vases utilisées pour cette étude proviennent de l'estuaire de la Seine (avant-port du Havre). Ce sont des matériaux sursaturés en eau et qui présentent une caractéristique essentielle très marquée : leur thixotropie. Cette caractéristique présente pour nous l'avantage de pouvoir considérer les vases prélevées comme retournées à leur état physique initial lorsqu'après prélévement in situ, nous les homogénéisons à nouveau, par malaxage, dans leur fût de stockage.

\subsection{Teneur en eau, limites d'Atterberg et densité}

Les teneurs en eau pondérale mesurées sont comprises entre 150 et $157 \%$. Ces mesures ont été effectuées par pesée avant et après passage à l'étuve à $105^{\circ} \mathrm{C}$. La présence de matières organiques, connue par les études antérieures, peut sans doute être considérée comme responsable de la variabilité des valeurs de teneur en eau observées malgré une bonne homogénisation du matériau avant prélévement.

Pour des raisons analogues, les limites de liquidité (wl) mesurées évoluent entre $62,3 \%$ et $65,1 \%$, les limites de plasticité ( $w_{p}$ ) entre $41,9 \%$ et $46,4 \%$. Ce qui nous donne un indice de plasticité $\left(\mathrm{I}_{\mathrm{p}}\right)$ compris entre $18,7 \%$ et $20,3 \%$. Cela aboutit à un indice de consistance $\left(\mathrm{I}_{\mathrm{C}}\right)$ inférieur à 2,4 .

Après homogénéisation, plusieurs mesures de masse volumique ont été réalisées sur des échantillons correspondant à un volume d'1,6 litre. Compte-tenu de la forte viscosité du matériau il a été difficile d'obtenir une mesure de volume très précise en raison d'états de surface non rigoureusement horizontaux. Cependant, il a été possible d'adopter une densité constante de 1,3 pour chacune des manipulations et préparations ultérieures.

\subsection{Analyse dimensionnelle}

Sur les échantillons de vase prélevés, une analyse dimensionnelle a été effectuée par tamisage sous eau. Le refus à $200 \mu \mathrm{m}$ est égal à $0,17 \%$, c'est-à-dire que la quasi totalité des échantillons a une dimension inférieure à $200 \mu \mathrm{m}$ $(99,83 \%)$. Les agrégats les plus grossiers (environ $100 \mu \mathrm{m}$ ) sont désagrégés. Mais 
compte-tenu des résultats précédents (Troalen, 1996, 1998) les pourcentages en argiles (sens granulométriques) et en argiles (sens minéralogique) sont assez difficilement comparables. Là encore, nous pouvons penser que la totalité des agrégats de particules fines n'a pas été mise en dispersion. Nous vérifions toutefois que l'essentiel de notre matériau est constitué par des particules et agrégats inférieurs à $100 \mu \mathrm{m}$.

\subsection{Analyse minéralogique}

Les minéraux constitutifs des vases ont été identifiés par diffractométrie aux rayons X (Marot, 1997 ; Troalen, 1996, 1998). Ainsi l'essentiel de la fraction minérale est constituée par du quartz (33\%), de la calcite (25\%) et par environ $36 \%$ de minéraux argileux (dont $20 \%$ de montmorillonite), la fraction restante pouvant être attribuée à des feldspaths (traces), à de la dolomite et du sel.

\section{Comportement mécanique de mélanges vase/coulis de ciment}

L'objectif de cette étude est de nous permettre de définir un mélange vase/coulis, avec des propriétés optimales tant du point de vue de la résistance mécanique obtenue après injection et réaction de prise, que du point de vue recyclage et donc utilisation du matériau initial, c'est-à-dire les vases de Seine.

\subsection{Protocole expérimental}

Dans toute l'étude nous avons pris comme repère trois mélanges comportant $25 \%, 50 \%$ et $75 \%$ de vase, le complément à $100 \%$ étant assuré uniquement par le coulis de ciment. Les mélanges vase/coulis sont effectués sur des matériaux de même densité, choisie égale à 1,3 (§ 2.1). Pour la préparation du coulis de ciment, l'équivalence de densité avec la vase a été obtenue à l'aide d'un abaque préexistant.

Du point de vue expérimental, deux séries d'essais ont été réalisées sur les mélanges vase/coulis. La première série consiste en une analyse de la réaction de prise par la mesure de la cohésion intacte non drainée à l'aide d'un scissomètre de laboratoire et d'un fall-cone. La seconde série correspond à des essais de compression uniaxiale sur les éprouvettes réalisées avec les différents mélanges et après un temps de repos (et de prise) de 7 jours. Cette seconde série d'essais nous permettait de mesurer la résistance maximale à la rupture et le module de Young du matériau fabriqué.

\subsection{Etude de la cohésion intacte non drainée}

En raison de la variabilité de la valeur de la cohésion intacte non drainée, variabilité due à la fois à la nature (pourcentages relatifs) des mélange et à la vitesse de la réaction de prise, l'expérience a montré qu'il était judicieux de mesurer cette cohésion au scissomètre lors des premières $48 \mathrm{~h}$ suivant la préparation du mélange. Passé $48 \mathrm{~h}$, les échantillons étant devenus trop cohérents, la mesure de la cohésion a été réalisée à l'aide du fall-cone (ou cône de pénétration) utilisé principalement pour mesurer une limite de liquidité équivalente à celle classiquement obtenue par les limites d'Atterberg. Des abaques permettent en effet de relier, pour un essai, la valeur de l'enfoncement à la valeur de la cohésion correspondante. 
Les principaux résultats sont présentés sur le tableau 1.

\begin{tabular}{|llllll|}
\hline $\mathrm{V}=$ vase & $\mathrm{C}=$ Coulis ciment & \multicolumn{4}{c|}{ temps de repos } \\
\hline mélange & nul & $1 \mathrm{~h}$ & $2 \mathrm{~h}$ & $24 \mathrm{~h}$ & $48 \mathrm{~h}$ \\
\hline $100 \% \mathrm{~V}$ & 0,21 & \multicolumn{5}{c|}{0,18} \\
\hline $75 \% \mathrm{~V} / 25 \% \mathrm{C}$ & 0,24 & 0,55 & 1,15 & 13,61 & 22,00 \\
\hline $50 \% \mathrm{~V} / 50 \% \mathrm{C}$ & 0,05 & 0,13 & 0,40 & 30,00 & 115,0 \\
\hline $25 \% \mathrm{~V} / 75 \% \mathrm{C}$ & 0,03 & 0,05 & 0,05 & 27,30 & 85,00 \\
\hline $100 \% \mathrm{C}$ & 0 & 0 & 0,05 & & \\
\hline appareillage & scissomètre & & & fall-cone \\
\hline
\end{tabular}

Tableau 1 : Valeurs de la cohésion intacte non drainée $(\mathrm{kPa})$

Table 1 : Values of the natural undrained cohesion $(\mathrm{kPa})$

En ce qui concerne les mesures de la cohésion sur la vase seule (tableau 1), la valeuir à $2 \mathrm{~h}$ apparaît comme plus faible $(0,18 \mathrm{kPa})$ que celle mesurée immédiatement après le mélange $(0,21 \mathrm{kPa})$. Ce comportement doit être attribué au caractère thixotropique des vases. Celles-ci reprennent leur structure initale, c'est-à-dire avant homogénisation de l'échantillon. Les teneurs en eau effectuées au temps 0 (w $=160,9 \%)$ et à $2 h(w=158,1 \%)$ ne peuvent pas être considérées comme significatives, malgré une légère baisse logiquement attribuée à un début de séchage. D'autre part, il convient d'être très prudent lorsque nous mesurons au scissomètre de très faibles valeurs angulaires, c'est-à-dire lorsque nous sommes dans le domaine des très faibles valeurs de cohésion.

Ces remarques étant faites, nous constatons que les valeurs de la cohésion intacte non drainée, pour un mélange donné, croissent avec le temps de repos. Cette évolution semble logique puisque dans le temps se développent deux phases en parallèle : séchage et réaction de prise. Par contre, si nous remarquons que la valeur la plus élevée de la cohésion intacte non drainée $(115 \mathrm{kPa}, \mathrm{w}=141 \%)$ est obtenue à $48 \mathrm{~h}$ pour le mélange $50 \%$ Vase $/ 50 \%$ Coulis, les réactions de prise semblent plus rapides pour les mélanges contenant le pourcentage de vase le plus élevé.

Il est évident que la vitesse de prise est, tout comme la valeur de la cohésion intacte non drainée, en relation directe avec la quantité d'eau disponible dans le mélange. Dans le cas des essais réalisés, l'acquisition d'une cohésion "acceptable" se réalise entre une teneur en vase comprise entre $75 \%$ et $50 \%$, à partir d'un temps de repos proche de $24 \mathrm{~h}$ et pour une perte en eau d'environ $30 \%$ par rapport à la teneur en eau initiale. Le processus précis de cette acquisition des propriétés mécaniques par les mélanges vase/coulis mériterait une étude plus approfondie, d'une part en effectuant des mesures de la cohésion à des intervalles de temps plus court, d'autre part, en utilisant des mélanges avec des teneurs vase/coulis intermédiaires. Dans le cas présent, les mesures de cohésion effectuées étaient réalisées comme indicateur des réactions de prise lors de la fabrication parallèle des éprouvettes vase/coulis destinées aux essais de compression uniaxiale. Il était effectivement impossible d'effectuer ces mesures sur les éprouvettes mêmes durant leur temps de repos d'une semaine. 


\subsection{Etude de la résistance maximale à la rupture}

L'objectif est d'évaluer la résistance maximale des différents mélanges vase/coulis de ciment, d'une manière telle que ces mélanges présentent des propriétés mécaniques appréciables et que la vase y soit en quantité suffisante pour être de cette façon "recyclée" massivement.

\subsubsection{Protocole expérimental}

Des éprouvettes cylindriques d'élancement égal à 2 ont été fabriquées à partir des différents mélanges vase/coulis en utilisant des moules en carton préalablement parafinés afin d'éviter toute perte en eau autre que celle liée à la réaction de prise et à la durée du séchage (temps de repos égal à 7 jours). 3 éprouvettes ont été fabriquées pour chacun des mélanges et également pour la vase $(100 \% \mathrm{~V})$ et le coulis de ciment $(100 \% \mathrm{C})$ seuls. Les teneurs en eau initiales sont mesurées. Après un repos en enceinte climatisée, les éprouvettes sont démoulées et rectifiées (dimensions exactes : hauteur $98 \mathrm{~mm}$, diamètre $45 \mathrm{~mm}$ ).

\subsubsection{Essais de compression uniaxiale}

La teneur en eau initiale moyenne des éprouvettes était d'environ $160 \%$. Après démoulage et rectification des éprouvettes, nous avons constaté des aspects de surface distincts. En effet, une famille d'éprouvettes présentait une texture externe "granuleuse et sèche" et correspondait à des teneurs en eau finales faibles; les essais de compression correspondant seront par la suite désignés sous le terme "d'essais à faible teneur en eau". A l'opposé, une autre famille d'éprouvettes présentait une texture "moins franche et plus humide", pour des temps de prise analogues à ceux de la famille précédente. Ces éprouvettes possédaient des teneurs en eau finales élevées et les essais de compression seront ainsi désignés sous le terme "d'essais à forte teneur en eau". Pour les essais, il a été utilisée une presse asservie avec enregistrement automatique des paramètres et la vitesse de déplacement était de $0,5 \mathrm{~mm} / \mathrm{mn}$.

* Essais à faible teneur en eau:

En raison de l'état initial des éprouvettes après rectification et de la variabilité des élancements obtenus, nous avons été obligés de "normaliser" après essai les courbes de compression en tenant compte de l'élancement réel ramené à 2.

La figure 1 donne les résultats des essais de compression uniaxiale obtenus sur les éprouvettes $50 \% \mathrm{~V}-50 \% \mathrm{C}$. En moyenne, la résistánce à la rupture $\mathrm{R}_{\mathrm{C}}$ est égale à $790 \mathrm{kPa}$ pour une déformation $\varepsilon=1,9 \%$ et un module de Young de 92 $\mathrm{MPa}$, avec une teneur en eau de $30,7 \%$ environ.

La figure 2 donne les résultats obtenus pour les trois éprouvettes contenant $25 \% \mathrm{~V}-75 \% \mathrm{C}$.

Les valeurs moyennes sont, pour la résistance maximale à la rupture $\left(\mathrm{R}_{\mathrm{C}}\right) 2400 \mathrm{kPa}$ et une déformation $\varepsilon=2,3 \%$, avec un module de Young de 160 MPA pour une teneur en eau de $28 \%$. Ces éprouvettes présentaient toutes un plan de fracture vertical particulièrement franc et ouvert de quelques $1 / 10 \mathrm{~mm}$ en fin d'essai.

* Essais à forte teneur en eau

Les éprouvettes $100 \% \mathrm{~V}$ ayant perdu très peu d'eau durant la période de repos ( $\mathrm{w}=127,7 \%$ ), il n'a pû être exécuté d'essais (tassement sous poids propre). Pour les éprouvettes $50 \% \mathrm{~V}-50 \% \mathrm{C}$, les résultats des essais de compressions sont reportés sur la figure 3. 
$\mathrm{R}_{\mathrm{C}}$ a une valeur d'environ $540 \mathrm{kPa}$ à $\varepsilon=2,9 \%$, avec un module de Youg $E=63,8$ MPa et une teneur en eau de $111 \%$.
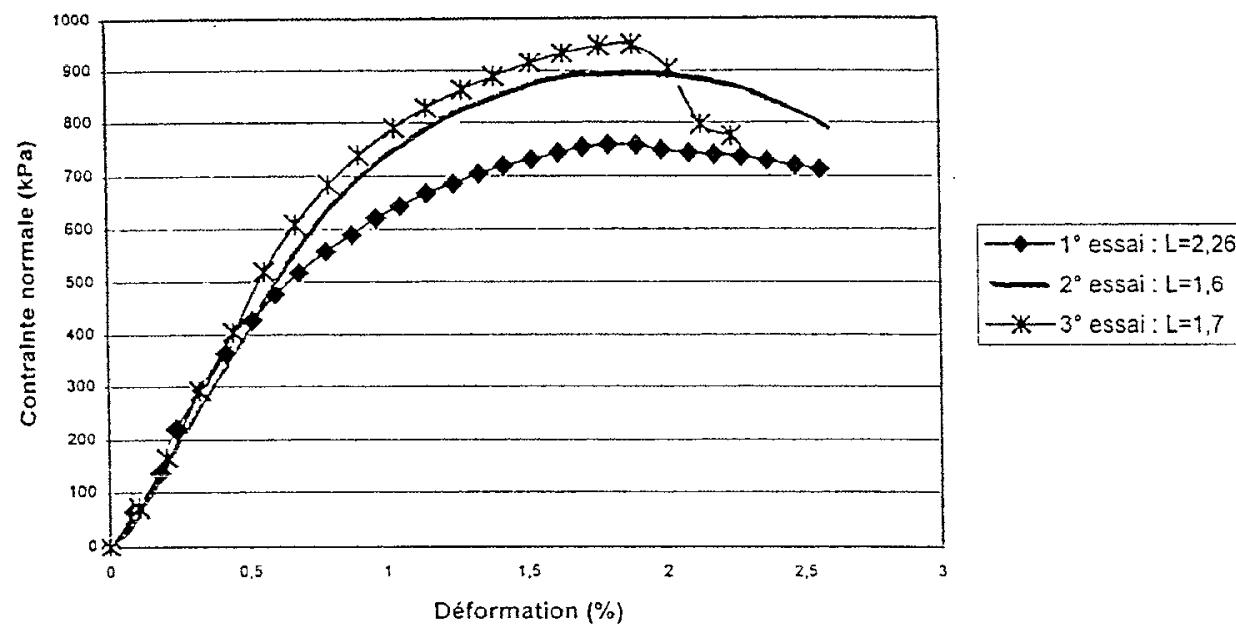

Figure 1 : Essais de compression uniaxiale, $50 \% \mathrm{~V}-50 \% \mathrm{C}$, w faible.

Figure 1 : Uniaxial compression tests, $50 \% \mathrm{~V}-50 \% \mathrm{C}$, low w.
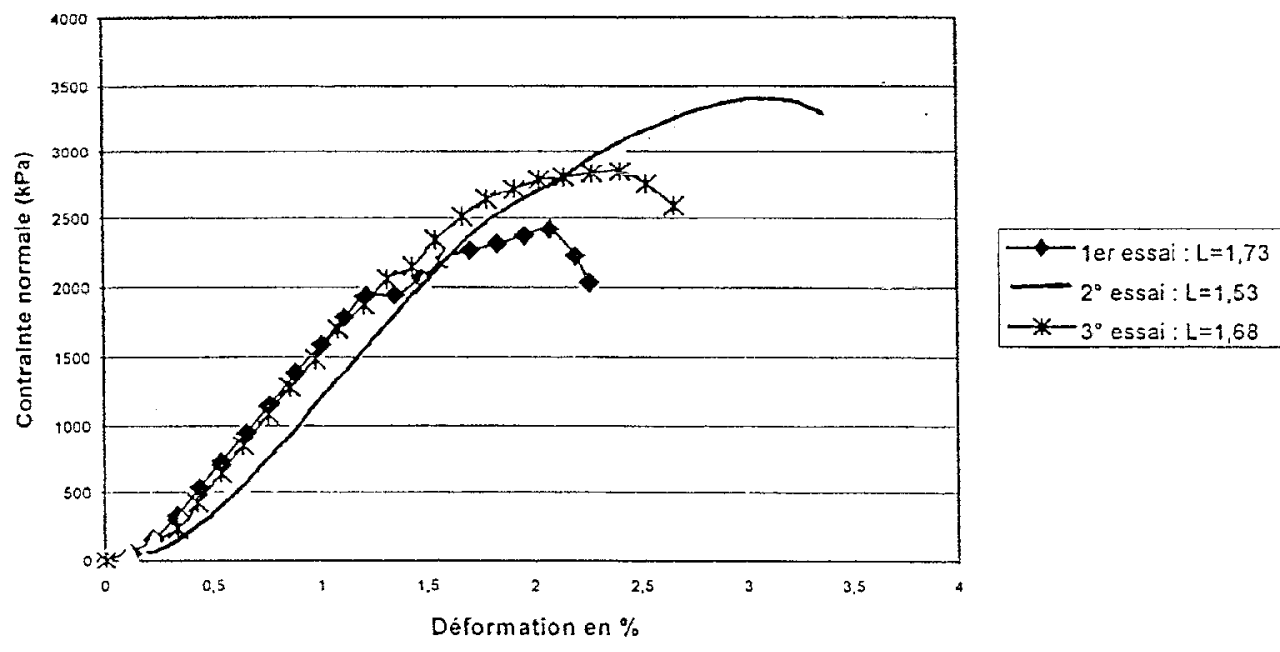

Figure 2 : Essais de compression uniaxiale, $25 \% \mathrm{~V}-75 \% \mathrm{C}$, w faible.

Figure 2 : Uniaxial compression tests, $25 \% \mathrm{~V}-75 \% \mathrm{C}$, low w.

Les courbes contrainte-déformation présentent une phase importante de déformation croissante à contrainte constante (Figure 3 ). Cette phase peut être interprétée comme un phénomène d'écrouissage. La présence d'une forte teneur en eau dans les éprouvettes lutte contre les processus de fracturation nette au profit d'un phénomène de propagation lente des discontinuités. En ce qui concerne les éprouvettes fabriquées avec un mélange $25 \% \mathrm{~V}-75 \% \mathrm{C}$, la forte teneur en eau $(108,8 \%)$ n'a pas permis d'obtenir plus d'une éprouvette testable. 


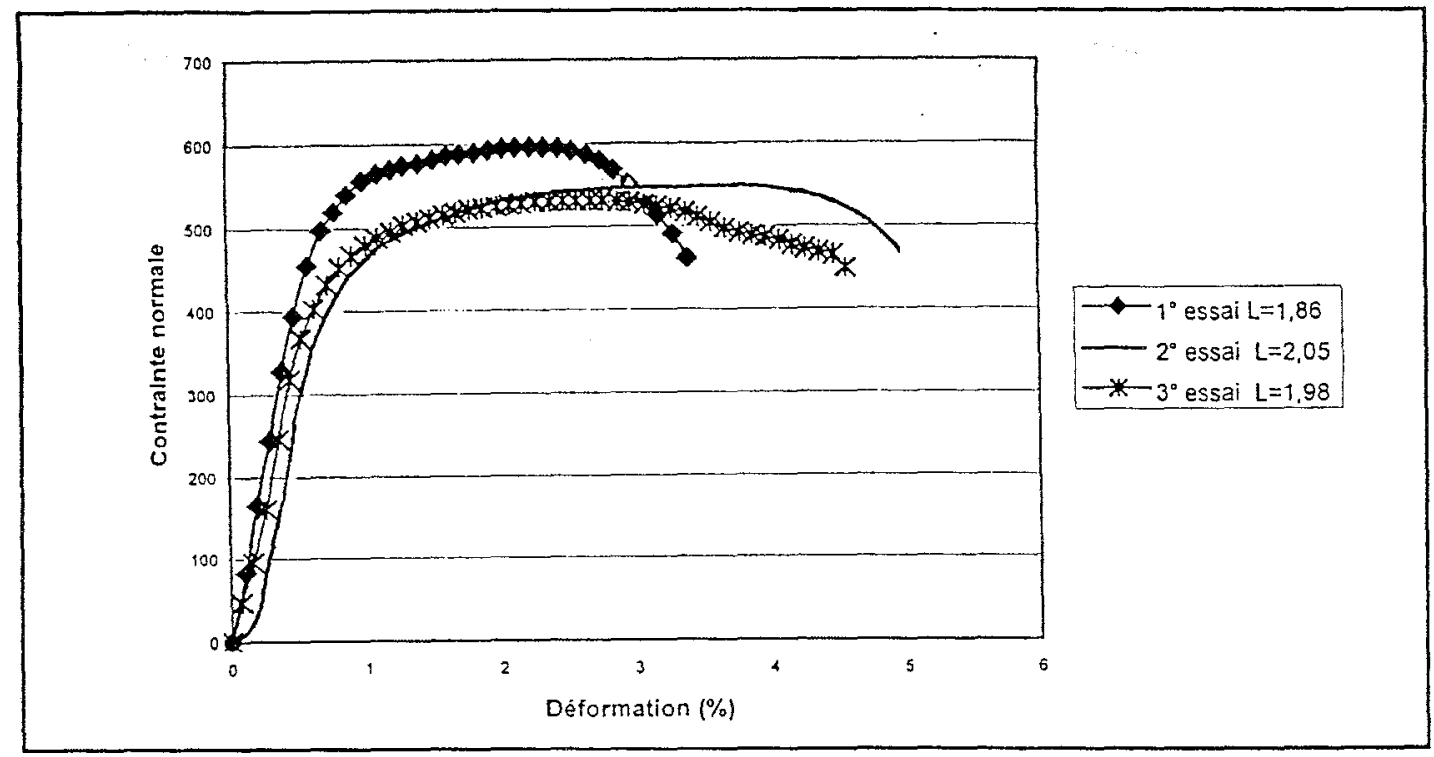

Figure 3 : Essais de compression uniaxiale, $50 \% \mathrm{~V}-50 \% \mathrm{C}$, w forte.

Figure 3 : Uniaxial compression tests, $50 \% \mathrm{~V}-50 \%$ c, high $w$.

Cet unique résultat donnait pour $\mathrm{R}_{\mathrm{C}}$ une valeur de $525 \mathrm{kPa}$ à $\varepsilon=2 \%$, avec $\mathrm{E}$ $=73 \mathrm{MPa}$. Cette valeur de Rc apparaît faible, mais l'éprouvette obtenue était de bonne qualité et l'expérience a montré que les éprouvettes de chaque lot présentaient des résultats cohérents. Ce résultat peut donc être considéré comme fiable.

Dans le cas des éprouvettes $100 \% \mathrm{C}$, un retrait très important était observé à l'issue du temps de repos (7 jours). $\mathrm{Rc}=3750 \mathrm{kPa}$ à $\varepsilon=3,8 \%$, avec $\mathrm{E}=100 \mathrm{MPa}$ et une teneur en eau de $56,2 \%$, assez surprenante quant à l'aspect externe présenté avant mesure. Cette série est donc à rapprocher des essais à faible teneur en eau.

\section{Discussion}

L'analyse des résultats montre que les familles d'essais n'ont pas subi les même processus de perte en eau durant la période de repos (réaction de prise). Quand l'eau est en forte proportion dans les mélanges, elle atténue les écarts entre les valeurs de la résistance en compression due à la présence de vase. Il en est de même pour les modules de Young. Pour les deux mélanges comparables (à $50 \%$ et $25 \%$ de vase), les propriétés mécaniques obtenues sont en effet peu différentes à forte teneur en eau. L'évolution dans le temps va accentuer les différences de composition des mélanges. Elle accroît nettement les valeurs de Rc et de E. Malgré les nombreuses incertitudes qui subsistent quant à la précision des mesures, nous pouvons dire que ces résultats sont de bons indicateurs du comportement des mélanges $\mathrm{V}-\mathrm{C}$, tant du point de vue du court terme (mise en place/préparation au niveau du chantier : essais scissométriques, § 3.2) que du moyen terme (propriétés mécaniques $\mathrm{V}-\mathrm{C}$ à 7 jours). Ce sont sur ces qualités finales qu'il nous faudra maintenant porter notre attention, en particulier sur leur évolution à long terme (influence du type de ciment, influence de minéraux initiaux tels que le sel, etc.). Un des principaux problèmes réside malgré tout dans le fait que la vase intacte est 
un matériau thixotropique visqueux et que sa forte teneur en eau (160\%) rend délicat sa valorisation immédiate (l'eau contenue s'échappe peu rapidement en milieu confiné). A l'inverse le coulis de ciment est au départ très liquide et possède des propriétés mécaniques nulles. En raison de sa forte teneur en eau (180\%), le phénomène de prise est retardé dans le temps.

Ainsi, les mélanges V-C reflètent une double évolution des caractéristiques mécaniques : Les mesures de cohésion semblent indiquer une influence négative de la vase sur les propriétés mécaniques jusqu'à une teneur en vase supérieure à $50 \%$. Un rapport de 5 existe pour la cohésion intacte non drainée entre le mélange $50 \% \mathrm{v}$ $50 \% \mathrm{C}$ et le mélange $75 \% \mathrm{~V}-25 \% \mathrm{C}$. En ce qui concerne la résistance en compression uniaxiale et les modules de Young, pour des teneurs en eau faibles (caractéristiques finales des mélanges), l'influence négative de la vase est très forte au delà des $25 \%$ de vase ; en effet, il existe un rapport de 4 entre les valeurs des résistances en compression des mélanges $25 \% \mathrm{~V}-75 \% \mathrm{C}$ et $50 \% \mathrm{~V}-50 \% \mathrm{C}$.

Ainsi nous pouvons grossièrement définir une zone d'études à développer entre $25 \%$ et $75 \%$ de vase, notamment en considérant un ajout progressif égal à $10 \%$.

D'autre part, il faut noter que si la vase réduit fortement les qualités du coulis de ciment pur, il faut également penser à concevoir spécifiquement les mélanges en fonction du but recherché. Dans le cas d'une réutilisation en coulis d'injection, il est évident qu'il faudra tenir compte de l'influence de la viscosité puisque celle-ci diffère selon les proportions des mélanges. Une injection sera d'autant plus difficile que le mélange sera riche en vase. De plus, même si une partie de l'eau du mélange participe à la réaction de prise, l'exédent d'eau doit pouvoir s'évacuer dans le milieu (poreux ou fracturé) où s'effectue l'injection.

Il ne faut pas non plus oublier que cette étude est un travail préliminaire et que d'autres résultas sont encore à attendre dans les recherches actuellement en cours.

\section{Références}

MAROT F. (1997) - Caractérisation et traitement de sédiments de dragage contenant des polluants métalliques, Thèse de l'Université du Havre, France, 330p. TROALEN JP., DURON C. \& HAGNERE C (1996) - Traitement de vases de l'estuaire de la Seine, Revue Française de Géotechnique, 77, pp.31-36.

TROALEN JP. (1997) - Amélioration et densification de vases d'estuaire, Process of the $3^{\text {rd }}$ International Conference on Ground Improvment Geosystem, Londres (U.K.) 3-6/06/97, Thomas Telford publishing.

TROALEN JP. (1998) - Vases issues du dragage : solidification et comportement, Actes du Colloque MAGI'50 "Les matériaux argileux et la Géologie de l'Ingénieur", ENSG-Nancy (France), 21-22/09/98, pp.77-84, Sciences de la Terre/ASGA Editeur, ISBN 2-85555-053-X.

TROALEN JP. (1998) - Comportement et réutilisation de vases d'estuaire (Seine) traitées par un limon quaternaire, Process of the $8^{\circ}$ Congress of the Internat.Assoc. of Eng. Geol.and Environ., Vancouver (Canada), 21-25/09/98, pp. 3875-3882, Balkema, Rotterdam, ISBN 90-5410-990-4. 